\title{
A COUNTEREXAMPLE TO THE CONJECTURE OF WOESS ON SIMPLE RANDOM WALKS ON TREES
}

\author{
KENNETH A. BERMAN AND MOKHTAR KONSOWA
}

(Communicated by William D. Sudderth)

\begin{abstract}
Let $T$ be a locally finite tree with a countable number of vertices. The volume of $T$ is the energy dissipation of the unit flow from the root of infinity that divides equally at every branching of the tree. It follows from Thomson's Principle that if $T$ contains an infinite leafless subtree whose volume is finite then $T$ is transient. Woess [6] conjectured that the converse is also true. In this paper we give a counterexample to this conjecture by constructing a transient tree, such that every infinite leafless subtree has infinite volume.
\end{abstract}

Consider a locally finite tree $T$ with a countable set of vertices $V$ and edges $E$. We distinguish a reference (root) vertex of $T$, denoted by $r$. A leaf is a vertex of degree one which is different from the root. We assume that $T$ is leafless, i.e. does not contain any leaves. When talking of infinite subtrees, we implicitly mean leafless infinite subtrees. For $v \in V$ let $d(v)$ denote the degree of $v$. A simple random walk (SRW) on $T$ is the Markov chain $X_{n} \quad(n=$ $0,1, \ldots)$ such that the state space of $\left(X_{n}\right)$ is $V$ and the one step transition probabilities $\operatorname{Pr}\left[X_{n+1}=v \mid X_{n}=u\right]=p_{u v}(T)(u, v \in V)$ are given by

$$
p_{u v}(T)= \begin{cases}1 / d(u) & \text { if } v \text { is adjacent to } u \\ 0 & \text { otherwise. }\end{cases}
$$

Thus if we are at vertex $u$ there is an equal probability of moving to any of its neighbors when we take the next step. Let $p_{u v}^{(n)}(T)$ denote the probability of being at vertex $v$ after $n$ steps having started from vertex $u$, i.e.

$$
p_{u v}^{(n)}(T)=\operatorname{Pr}\left[X_{n}=v \mid X_{0}=u\right] .
$$

Let $G_{u v}(T)$ be the mean number of visits to vertex $v$ during the SRW after starting at $u$. It is immediate that

$$
G_{u v}(T)=\sum_{n=0}^{\infty} p_{u v}^{(n)}(T) .
$$

Received by the editors June 12, 1987 and, in revised form, March 4, 1988.

1980 Mathematics Subject Classification (1985 Revision). Primary 60J15; Secondary 05C05. 
One of the most important concepts concerning Markov chains is that of recurrence and transience. The tree $T$ is called

$$
\begin{aligned}
& \text { recurrent, if } G_{u v}(T)=\infty, \\
& \text { transient, if } G_{u v}(T)<\infty .
\end{aligned}
$$

This definition does not depend on the particular choice of $u, v \in V$ [1]. Thus $T$ is transient iff $G_{r r}(T)<\infty$ where $r$ is the root vertex. Necessary and sufficient conditions for recurrence of a reversible Markov chain (of which a simple random walk is a special case) have been given by Nash-Williams [5] and Lyons [4]. A simple condition for recurrence of simple random walks on trees was conjectured by Woess [6]. In this paper we give a counterexample to Woess' conjecture. Before stating the conjecture we establish some terminology and state some well-known results about $G_{r r}(T)$ which we employ in constructing the counterexample.

Consider a tree $T$ which is finite. We will regard the leaves of $T$ as absorbing states, i.e. a node $u$ which is not a leaf obeys (1) but a node $u$ which is a leaf satisfies

$$
p_{u v}(T)= \begin{cases}1 & \text { if } u=v \\ 0 & \text { otherwise }\end{cases}
$$

Then $p_{r r}^{(n)}(T)$ is the probability of starting at vertex $r$ and returning to $r$ after $n$ steps without ever having visited a leaf. Further $G_{r r}(T)$ is the mean number of visits to vertex $r$ without ever having visited a leaf. Let.

$$
R(T)=G_{r r}(T) / d(r) .
$$

There is a special interpretation for $R(T)$ in electrical network theory. Let $T^{\prime}$ be the graph obtained from $T$ by identifying all the leaves of $T$ to form a single vertex $s$. If $T^{\prime}$ is regarded as an electrical network with source $r$ and sink $s$ where all the edges have resistance 1 then $R(T)$ is the effective resistance of $T^{\prime}$. See [2].

Proposition 1. Let $T$ be a tree consisting of two subtrees $S_{1}$ and $S_{2}$ having only the root $r$ in common. Then

$$
1 / R(T)=1 / R\left(S_{1}\right)+1 / R\left(S_{2}\right) .
$$

Proof. Let $S_{1}^{\prime}$ and $S_{2}^{\prime}$ be the two subgraphs of $T^{\prime}$ corresponding to $S_{1}$ and $S_{2}$, respectively. Then $S_{1}^{\prime}$ and $S_{2}^{\prime}$ are connected in parallel and Proposition 1 follows immediately from the Parallel Law of electricity. See [2].

A path is a connected graph $P$ such that every vertex of $P$ has degree two except for two vertices (the ends of $P$ ) which have degree one.

Proposition 2. Let $T$ be a tree consisting of a path $P$ of length $k$ joining the root $r$ to vertex $u$ and a tree $S$ such that $P$ and $S$ have only the vertex $u$ in common. Then

$$
R(T)=k+R(S)
$$


Proof. Let $P^{\prime}$ and $S^{\prime}$ be the path and subtree of $T^{\prime}$ corresponding to $P$ and $S$ in $T$. Then $P^{\prime}$ and $S^{\prime}$ are connected in series and Proposition 2 follows from the Series Law of electricity with the observation that the effective resistance of a path of length $k$ is $k$. See [2].

Proposition 3. Let $T^{m}$ be the tree obtained from $T$ by subdividing every edge of $T$ into $m$ small edges, i.e. replacing every edge by a path of length $m$. Then

$$
R\left(T^{m}\right)=m R(T) .
$$

Proof. Replacing an edge by $m$ edges connected in series is equivalent to increasing the resistance of that edge to $m$. Thus replacing every edge by $m$ edges connected in series increases the effective resistance of $T^{\prime}$ by a factor of $m$.
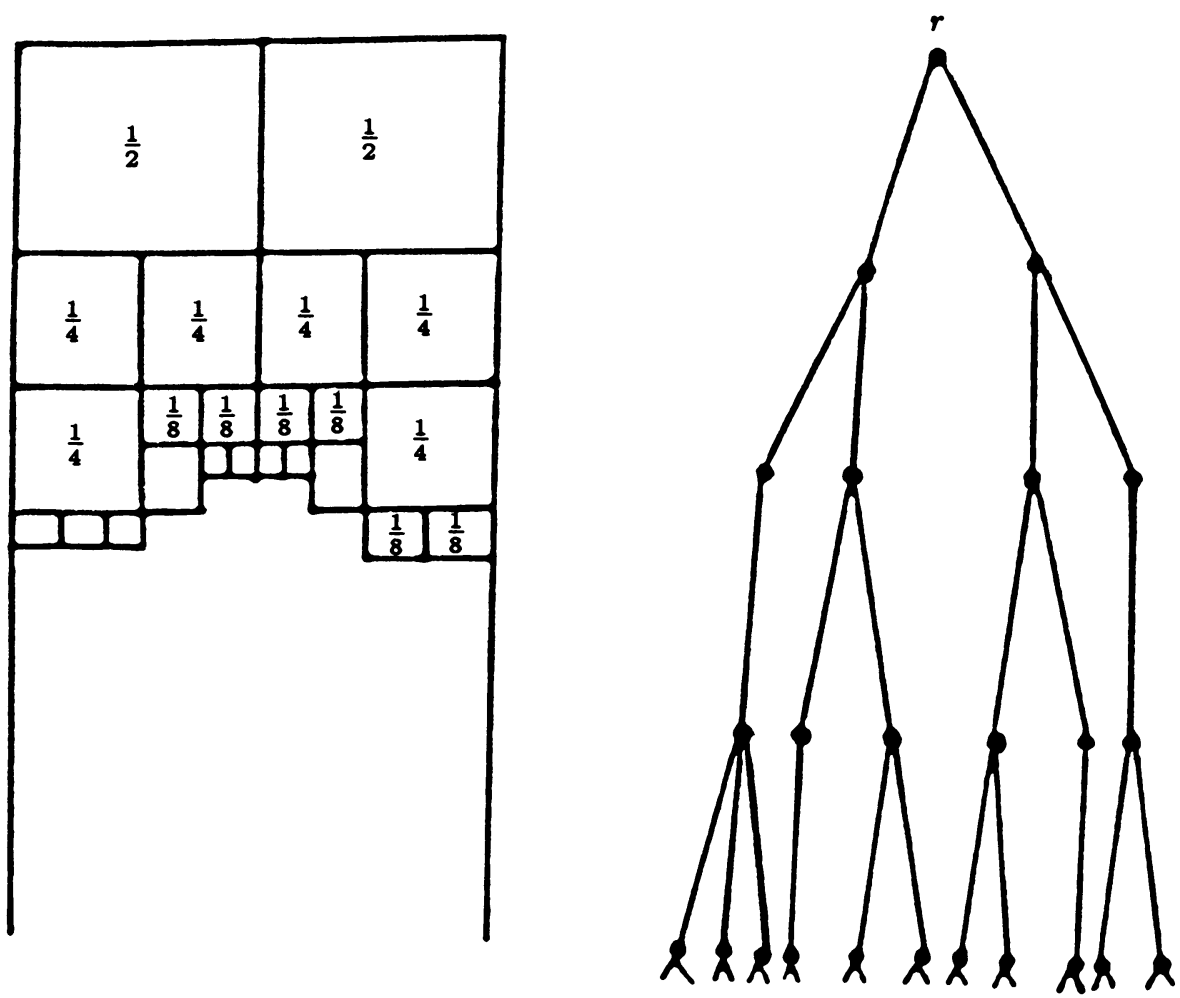

Figure 1

Proposition 4. Let $T$ be a tree with a countably infinite number of vertices. For each positive integer $i$ let $T_{i}$ be a finite subtree which contains every edge $e$ of $T$ such that both end vertices of $e$ have distance at most $i$ from the root vertex $r$. Then

$$
G_{r r}(T)=\lim _{i \rightarrow \infty} G_{r r}\left(T_{i}\right) .
$$


Proof. For $H$ a tree let $s_{i}(H)=\sum_{n=0}^{i-1} p_{r r}^{(n)}(H)$. For $n<i, p_{r r}^{(n)}(T)=p_{r r}^{(n)}\left(T_{i}\right)$ since a walk in $T_{i}$ starting at $r$ can never reach a leaf in only $n$ steps. Thus

$$
s_{i}\left(T_{i}\right)=s_{i}(T)
$$

Clearly, $s_{i}\left(T_{i}\right) \leq G_{r r}\left(T_{i}\right)$. Thus,

$$
G_{r r}(T)=\lim _{i \rightarrow \infty} s_{i}(T)=\lim _{i \rightarrow \infty} s_{i}\left(T_{i}\right) \leq \lim _{i \rightarrow \infty} G_{r r}\left(T_{i}\right) .
$$

But $G_{r r}(T) \geq G_{r r}\left(T_{i}\right)$ for all positive integers $i$. Hence,

$$
G_{r r}(T) \geq \lim _{i \rightarrow \infty} G_{r r}\left(T_{i}\right)
$$

This proves Proposition 4.

We now define the volume of a (finite or infinite) tree $T$ as follows. (See $[3,6]$.) Orient the edges of $T$ such that there is a directed path from the root vertex $r$ to all the other vertices. Let $d^{+}(v)$ denote the outdegree of vertex $v$, i.e. the number of edges with tail $v$. Let $t(e)$ denote the tail of edge $e \in E$. Assign to each edge $e \in E$ the weight $w(e)=w_{T}(e)$ given by

$$
w(e)=1 / d^{+}(t(e)) \text {. }
$$

Let $\mathscr{P}_{r}=\mathscr{P}_{r}(T)$ denote the set of all paths in $T$ whose initial vertex is $r$. The volume of $T$ denoted by $\mathscr{V}_{r}$ is defined by

$$
\mathscr{V}_{r}=\sum_{P \in \mathscr{P}_{r}} \prod_{e \in P} w^{2}(e)
$$

where the summation is over all finite paths $P \in \mathscr{P}_{r}$ and the product is over all the edges $e$ in $P$. See Figure 1 .

The volume is exactly the energy dissipation of the unit flow from the root to infinity that divides equally at every branching of the tree. This follows immediately from the definitions of a unit flow and energy dissipation. We refer the reader to [2] for these definitions. Thomson's Principle characterizes the effective resistance of a network as the minimum energy dissipation among all unit flows through the network (see [2, pp. 63-64]). This makes it obvious that a tree is transient if it contains a subtree with finite volume. Woess [6] conjectured that the converse is also true. We give a counterexample to this conjecture by constructing a transient tree, all of whose infinite subtrees have infinite volume.

The set of vertices adjacent to a vertex $v$ of a tree $T$ which are further from the root than $v$ are called the children of $v$. If $v$ has exactly two children we will refer to one child as the left child and the other as the right child. Consider the complete binary tree $C_{i}$ with $i$ levels, i.e. every vertex which is not a leaf has exactly two children and any path from the root to a leaf has length $i$. Label the left and right children of the root $r$ with the labels (0) and (1), respectively. Inductively, label a vertex $\left(x_{1}, \ldots, x_{k}, 0\right)$ if it is the left child of the vertex labeled $\left(x_{1}, \ldots, x_{k}\right)$ and $\left(x_{1}, \ldots, x_{k}, 1\right)$ if it is the right child. Label an edge 


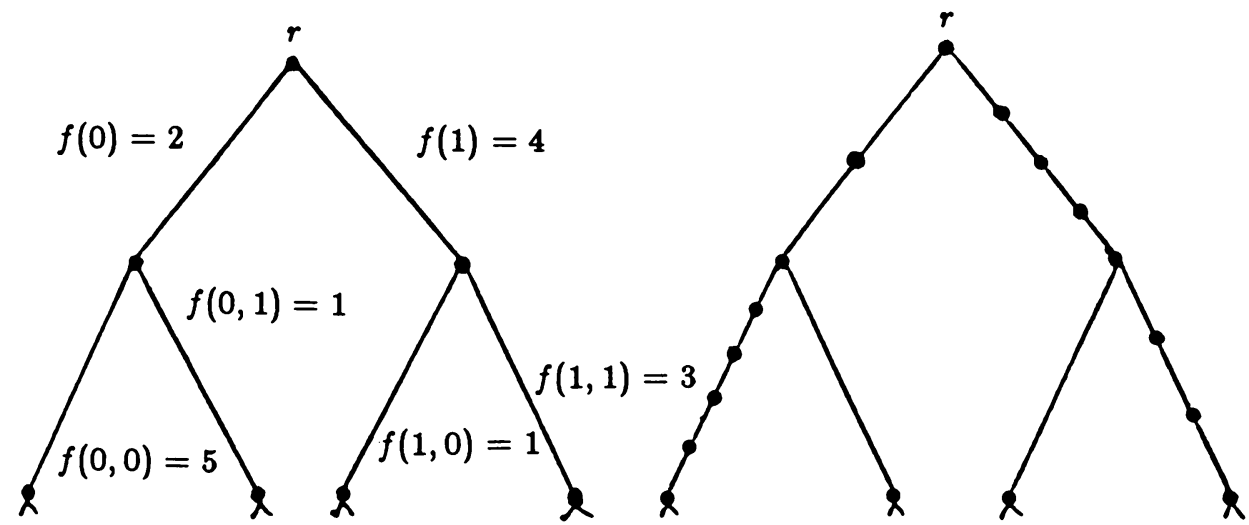

FIGURE 2

$e\left(x_{1}, \ldots, x_{k}\right)$ if $\left(x_{1}, \ldots, x_{k}\right)$ is the label of the vertex of $e$ further from the root. Let $L$ denote the set of all labels, i.e.,

$$
L=\bigcup_{k=1}^{i}\{0,1\}^{k}
$$

Consider a mapping $f$ from $L$ to the positive integers. Let $C_{i}(f)$ be the tree obtained from $C_{i}$ by replacing each edge $\left(x_{1}, \ldots, x_{k}\right)$ with a path of length $f\left(x_{1}, \ldots, x_{k}\right)$. See Figure 2. For $m$ any positive integer let $T_{1}(m)$ be the tree $C_{i}(f)$ such that

$$
f\left(x_{1}, \ldots, x_{k}\right)=m^{q} \text { where } q=x_{1}+\cdots+x_{k} .
$$

See Figure 3. Let $T(m)$ be the (infinite) tree obtained by taking the limit of $T_{i}(m)$ as $i$ goes to infinity, i.e.,

$$
T(m)=\lim _{i \rightarrow \infty} T_{i}(m)
$$

Theorem 5. Let $T_{i}(m)$ be defined as above. Then

$$
G_{r r}\left(T_{i}(m)\right)=2 m\left(1-(m /(m+1))^{i}\right) .
$$

Proof. Let $e_{1}$ and $e_{2}$ denote the edge joining $r$ to its left child and right child, respectively. Let $S_{1}$ and $S_{2}$ be the left and right subtrees of the root $r$, i.e. the branches of $T_{i}(m)$ containing edges $e_{1}$ and $e_{2}$, respectively. Let $u$ denote the vertex of $e_{1}$ different from $r$, i.e. the left child of $r$. Then $S_{1}$ consists of the edge $e_{1}$ together with a subtree rooted at $u$ which is isomorphic to $T_{i-1}(m)$. Thus by Proposition 2

$$
R\left(S_{1}\right)=1+R\left(T_{i-1}(m)\right) \text {. }
$$

Now $S_{2}$ is isomorphic to the tree obtained from $S_{1}$ by replacing every edge of $S_{1}$ with a path of length $m$. Therefore, by Proposition 3

$$
R\left(S_{2}\right)=m R\left(S_{1}\right) \text {. }
$$


By Proposition 1, we have

$$
1 / R\left(T_{i}(m)\right)=1 / R\left(S_{1}\right)+1 / R\left(S_{2}\right) .
$$

Combining (14), (15), (16) and simplifying we obtain

$$
R\left(T_{i}(m)\right)=m /(m+1)\left(1+R\left(T_{i-1}(m)\right) .\right.
$$

It is easily verified that $R\left(T_{1}(m)\right)=m /(m+1)$. It follows by induction that

$$
R\left(T_{i}(m)\right)=\sum_{n=1}^{i}(m /(m+1))^{n}=m\left(1-(m /(m+1))^{i}\right) .
$$

Theorem 5 now follows immediately from (6).

Corollary 6. The tree $T(m)$ is transient and

$$
G_{r r}(T(m))=2 m
$$

Corollary 6 follows immediately from Proposition 4 and Theorem 5.

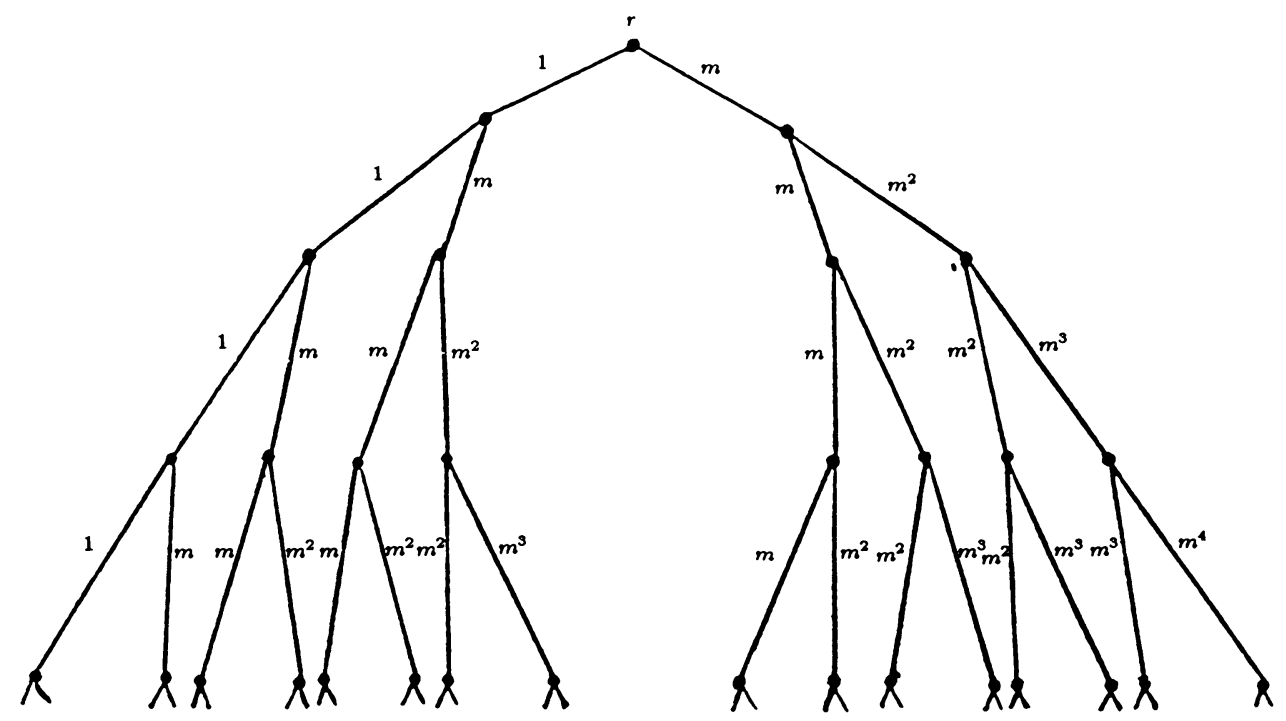

FiguRe 3

Theorem 7. Let $m$ be a positive integer, $m \geq 4$. Then any infinite subtree $S$ of $T(m)$ has infinite volume.

Proof. Let $r^{\prime}$ be the root of $S$, i.e. $r^{\prime}$ is the vertex of $S$ whose distance from $r$ is minimum. Let $Q=q_{0} q_{1} q_{2} \cdots$ be the infinite path in $S$ whose initial vertex is $r^{\prime}$, i.e. $q_{0}=r^{\prime}$, such that $q_{i+1}$ is the child of $q_{i}$ in the case when $q_{i}$ has only one child in $S$ and $q_{i+1}$ is the right child in the case when $q_{i}$ has two children in $S$. If only a finite number of vertices of $Q$ have two children in $S$ then $S$ must contain a branch which is a path of infinite length and therefore must 
have infinite volume. Thus we may assume that there is an infinite number of vertices of $Q$ which have two children in $S$. Let $q_{i_{0}}, q_{i_{1}}, q_{i_{2}} \cdots$ denote these vertices, where $i_{j-1}<i_{j}(j=1,2, \ldots)$. It follows easily from the definition of $T_{i}(m)$ that

$$
i_{j}-i_{j-1} \geq m^{j}
$$

Let $k$ denote the length of the subpath of $Q$ joining $r^{\prime}$ and $q_{i}$. Thus we have

$$
\begin{aligned}
\mathscr{V}_{r^{\prime}}(S) & =\sum_{P \in \mathscr{P}_{r^{\prime}}(S)} \prod_{e \in P} w^{2}(e) \quad \text { (by definition) } \\
& \geq \sum_{P \subset Q} \prod_{e \in P} w^{2}(e) \\
& =k+\sum_{j=1}^{\infty}(1 / 4)^{j}\left(i_{j}-i_{j-1}\right) \\
& \geq \sum_{j=1}^{\infty}(m / 4)^{j} \quad(\text { by }(19)) \\
& =\infty .
\end{aligned}
$$

This proves Theorem 7 .

Corollary 6 together with Theorem 7 show that $T(m)$ for all $m \geq 4$ is a counterexample to the conjecture of Woess. Now every infinite subtree of a recurrent tree must also be recurrent [6]. Thus if we add a recurrent branch to any vertex of a tree $T$ which is transient the new tree $\hat{T}$ must also be transient. Further if every infinite subtree of $T$ has infinite volume then every infinite subtree of $\hat{T}$ will also have infinite volume. This follows from the result [6] that a recurrent tree has infinite volume. Hence we can construct any number of transient trees all of whose subtrees have infinite volume by adding recurrent branches to $T(m)$ where $m \geq 4$.

\section{REFERENCES}

1. K. L. Chung, Markov chains with stationary transition probabilities, Grundlehren Math. Wiss., Band 104, Berlin-Heidelberg-New York, 1967.

2. P. Doyle and J. Snell, Random walks and electrical networks, The Carus Mathematical Monographs, Mathematical Society of America, 1984.

3. P. Gerl, Rekurrente und transient Baume, Seminaire Lotharingien de Combinatoire 10, IRMA, Strasbourg, 1984, pp. 80-87.

4. T. Lyons, A simple criterion for transience of a reversible Markov chain, Ann. Probab. 11 (1983), 393-402.

5. C. St. J. A. Nash-Williams, Random walks and electric currents in networks, Proc. Cambridge Philos. Soc. 55 (1959), 181-194.

6. W. Woess, Transience and volumes of trees, Arch. Math. 46 (1986), 184-192.

Department of Computer Science, Mail Location 8, 822 Old Chemistry Building, UniVERSity OF CincinNati, CincinNati, OHIO 45221-0008 